INPLASY

PROTOCOL

To cite: Wang et al. Clinical acupunture therapy for Femur Head Necrosis: protocol of a systematic review and metaanalyses. Inplasy protocol 202150035. doi:

10.37766/inplasy2021.5.0035

Received: 09 May 2021

Published: 10 May 2021

Corresponding author: Wenlong Yang

y378759501@163.com

Author Affiliation: The Affiliated Hospital of Jiangxi University of Traditional Chinese Medicine

Support: 2020ZGJO1.

Review Stage at time of this submission: The review has not yet started.

Conflicts of interest:

None declared.

\section{Clinical acupunture therapy for Femur Head Necrosis: protocol of a systematic review and meta-analyses}

Review question / Objective: Through a comprehensive review of literature, this study further systematically evaluated the efficacy and safety of acupuncture in the treatment of femoral head necrosis, with a view to clinical treatment of femoral head necrosis, alleviation of related clinical symptoms and prevention of its further development. And provide up-to-date evidence-based medicine evidence.

Information sources: Eight electronic databases, including the Cochrane Central Register of Controlled Trials, Web of Science, PubMed, EMBASE, China National Knowledge Infrastructure, Chinese Biomedical Literature Database, Technology Periodical database (Chinese Scientific Journal Database), and Wanfang Database.

INPLASY registration number: This protocol was registered with the International Platform of Registered Systematic Review and Meta-Analysis Protocols (INPLASY) on 10 May 2021 and was last updated on 10 May 2021 (registration number INPLASY202150035).

\section{INTRODUCTION}

Review question / Objective: Through a comprehensive review of literature, this study further systematically evaluated the efficacy and safety of acupuncture in the treatment of femoral head necrosis, with a view to clinical treatment of femoral head necrosis, alleviation of related clinical symptoms and prevention of its further development. And provide up-to-date evidence-based medicine evidence.

Rationale: Acupuncture and moxibustion therapy is widely used in patients who are reluctant to undergo primary total hip 
arthroplasty due to necrosis of the femoral head. The vigorous development and progress of acupuncture and moxibustion represents the development frontier of traditional Chinese medicine in treating non-surgical diseases. In this study, systematic review and network metaanalysis (NMA) were used to comprehensively evaluate the safety and effectiveness of acupuncture treatment for patients with necrosis of the femoral head caused by various reasons.

Condition being studied: Femur Head Necrosis(FHN) is a potentially devastating disease that is characterized by bone cells and bone marrow deaths, mainly caused by the lack of blood in the cartilage. This disease can occur at any age, but it is between 30 and 50 years old, and there is no difference. The main complaint of the FHN patients is most of the pain and blunt pain of the hip or peripheral joints, and the increase and severity of the activity will cause the joint activities to be limited. The range of pain is usually confined to the groin region, or in large, small, tuberous areas, where the pain is radioactive and radiates to the hips and knees. Weight increases pain, and rest relieves pain. This pain is described as a depth, intermittent, and sporadic pain. It may also be a sudden latent attack, which is gradually developing into persistent pain, stiff muscle and inability to autonomously. In the global range, FHN has a high risk of disability, which seriously affects people's health and quality of life. An epidemic school found that about 8.2 million Chinese people aged 15 and over were affected by the disease every year. Recent studies have shown that acupuncture has its unique advantages in treating the disease. In the case of surgical treatment of western medicine, acupuncture therapy has the advantages of regulating the Yin and Yang, preventing disease, the side effect and the acceptance of the patient.

\section{METHODS}

Search strategy: Systematic retrieval of MEDLINE, Web of Science, Scopus, Embase, Cochrane Library, Cumulative
Index of Nursing and Related Health Literature, and China's National Knowledge Infrastructure. We will search every electronic database from inception until May 1, 2020, with no language and publication date restrictions. This study will only consider randomized controlled trials that examine the efficacy and safety of acupuncture in patients with FHN. We will also establish a similar search strategy for other electronic databases. In addition, we will investigate other literature sources to avoid omission of potential studies, such as a reference list of conference abstracts and related reviews.

Participant or population: Femur Head Necrosis(FHN) is a potentially devastating disease that is characterized by bone cells and bone marrow deaths, mainly caused by the lack of blood in the cartilage. This disease can occur at any age, but it is between 30 and 50 years old, and there is no difference. The main complaint of the FHN patients is most of the pain and blunt pain of the hip or peripheral joints, and the increase and severity of the activity will cause the joint activities to be limited. The range of pain is usually confined to the groin region, or in large, small, tuberous areas, where the pain is radioactive and radiates to the hips and knees. Weight increases pain, and rest relieves pain. This pain is described as a depth, intermittent, and sporadic pain. It may also be a sudden latent attack, which is gradually developing into persistent pain, stiff muscle and inability to autonomously. In the global range, FHN has a high risk of disability, which seriously affects people's health and quality of life. An epidemic school found that about 8.2 million Chinese people aged 15 and over were affected by the disease every year. Recent studies have shown that acupuncture has its unique advantages in treating the disease. In the case of surgical treatment of western medicine, acupuncture therapy has the advantages of regulating the Yin and Yang, preventing disease, the side effect and the acceptance of the patient.

Intervention: Patients with FHN in the experimental group must be treated mainly 
with acupuncture (combined with other treatments or treated alone), and patients in the control group must not be treated with acupuncture.

Comparator: The control group can include blank control group, medicine (traditional Chinese medicine, western medicine) treatment, routine symptomatic treatment, etc. 1. Acupuncture treatment vs no treatment; 2. Acupuncture vs placebo; 3. Acupuncture versus symptomatic or active treatment.

Study designs to be included: Randomised controlled trial (RCT) study of acupuncture for FHN, published in any language.

Eligibility criteria: Types of study:All randomized controlled trials (RCT s) study on cupping therapy treatment of Intractable facial paralysis.Others such as case reports, animal experiments, non-RCTs, or RCT protocol will be excluded.

Information sources: Eight electronic databases, including the Cochrane Central Register of Controlled Trials, Web of Science, PubMed, EMBASE, China National Knowledge Infrastructure, Chinese Biomedical Literature Database, Technology Periodical database (Chinese Scientific Journal Database), and Wanfang Database.

Main outcome(s): The total effective rate.

Additional outcome(s): (1) Hip function (Hip Harris joint score, WOMAC hip score, hip joint Lequesne index score, Merle D 'Aubigne and hip joint Postel score); (2) Hip pain score (visual analogue Scale, VAS score); (3) Progress of imaging staging of femoral head necrosis Rate (staging method: ARCO, FICAT, Japanese Joint Association Staging); (4) volume percentage of femoral head necrosis; (5) Total hip replacement rate.

Quality assessment / Risk of bias analysis: Two reviewers performed rigorous methodological quality evaluation of the included studies with reference to the Cochrane Collaboration Bias Risk Assessment Tool for the extracted methodological features.

Strategy of data synthesis: Meta analysis was performed using RevMan5.3 provided by the Cochrane collaboration network.Relative risk (RR) was used for the two categorical variables, and mean difference(MD) was used for the continuous variables. Both were expressed with $95 \%$ confidence intervals $(\mathrm{Cl})$. The heterogeneity test between the results of the included studies was performed using the $I^{2}$ test.The $I^{2}$ value reflects the proportion of the total variation in the effect size due to the existence of heterogeneity. $\left(I^{2}>50 \%\right.$, indicating that heterogeneity is more obvious. If there is no obvious heterogeneity between the research results ( ${ }^{2} 50 \%$ ), the source of the heterogeneity is analyzed first, which may lead to heterogeneity Factors for subgroup analysis. If statistical heterogeneity exists in each subgroup without clinical heterogeneity, a random effects model is used for analysis. If the heterogeneity is too large and the results cannot be combined, a descriptive analysis is used and a sensitivity analysis is performed if necessary.

Subgroup analysis: Subgroup analysis will be handled according to the differences in Acupuncture methods, patient conditions, and control.

Sensitivity analysis: Sensitivity analyses will be performed to verify the robustness of the review conclusions. The impacts of study design, methodological quality, and missing data will be evaluated. Sensitivity analyses were planned by studies considered being at low risk of bias.

Language: No limited.

Country(ies) involved: China.

Keywords: acupuncture, Femur Head Necrosis, protocol, systematic review and meta-analysis. 
Contributions of each author:

Author 1 - Hongyu Wang.

Email: 2934786842@qq.com

Author 2 - Fengyun Yang.

Author 3 - Zhiwen Cao.

Email: 3082702175@qq.com 